\title{
Validity and reliability of the Oral Impacts on Daily Performance (OIDP) frequency scale: a cross-sectional study of adolescents in Uganda
}

\author{
Anne Nordrehaug Åstrøm*1,2 and Isaac Okullo1,3
}

\begin{abstract}
Address: ${ }^{1}$ Centre for International Health, Armauer Hansen Building, N-5021 Bergen, Norway, ${ }^{2}$ Department of Odontology-Community Dentistry, University of Bergen, Norway and ${ }^{3}$ Department of Dentistry, Makerere University, Uganda

Email: Anne Nordrehaug Åstrøm* - anne.nordrehaug@cih.uib.no; Isaac Okullo - isaac.okullo@student.uib.no

* Corresponding author
\end{abstract}

Published: 28 August 2003

BMC Oral Health 2003, 3:5

This article is available from: http://www.biomedcentral.com//472-683I/3/5

(C) 2003 Åstrøm and Okullo; licensee BioMed Central Ltd. This is an Open Access article: verbatim copying and redistribution of this article are permitted in all media for any purpose, provided this notice is preserved along with the article's original URL.
Received: 27 March 2003

Accepted: 28 August 2003

\begin{abstract}
Background: Assessing oral health related quality of life impact of mouth in adolescents is a relatively ignored area in dental research. This study aimed to examine reliability and validity of an abbreviated version of the oral impact of daily performance (OIDP) questionnaire and to analyse the interrelationship among OIDP scores, socio-demographic characteristics and oral health status in Uganda.
\end{abstract}

Method: II 46 adolescents (mean age 15.8, response rate 87\%) attending secondary schools in Kampala (urban) and Lira (rural) completed a survey instrument designed to measure subjective oral health indicators including the eight-item OIDP frequency scores. A clinical examination was conducted among 372 students (mean age 16.3, response rate $72 \%$ ) and caries was assessed following the World Health Organisation criteria (1997).

Results: $62 \%$ of the students experienced at least one oral impact during the 6 months preceding the survey. Cronbach's alpha for the OIDP frequency items was 0.91 and the corrected item-total correlation ranged from 0.62 to 0.75 . Discriminant and construct validity were demonstrated in that the OIDP scores varied systematically in the expected direction with missing teeth and selfreport indicators of oral health status, respectively. Socio-demographics and dental attendance did not predict OIDP through interaction with clinical indicators but varied systematically and independently with OIDP frequency scores in the multivariate analysis.

Conclusion: the OIDP frequency score have acceptable psychometric properties in the context of an oral health survey among Ugandan adolescents. Some evidence of the importance of social and personal characteristics in shaping adolescents' responses to oral disorders was provided.

\section{Background}

In response to the growing recognition of quality of life measurement in health care, socio-dental indicators, designed to assess the functional and psychological outcomes of oral disorders, have been developed and tested in various populations $[1,2]$. Most of the research on oral 
health related quality of life has been performed with adults and older people and there are only few studies from outside North-America and Europe $[1-3,5,6]$. Uncertainty remains as to the use of socio-dental indicators in youth populations generally and to their applicability in non-western cultural settings, specifically.

The Oral Impacts on Daily Performance (OIDP) scale [5] assesses impacts that affect individuals' daily life. Considering respondent burden, this instrument is advantageous for use in population surveys, not only in terms of being easier whilst measuring behaviours rather than feeling states, but also in being short. It is based on an explicit conceptual framework, the World Health Organisation's International Classification of Impairments, Disabilities and Handicaps, ICIDH [7], which has been amended for dentistry by Locker [8]. The ICDIH provides a basis for the empirical exploration of the links between different dimensions or levels of consequence variables and consists of the following key concepts; impairments, functional limitations, pain and discomfort and disability and handicap. Impairments refer to the immediate biophysical outcomes of disease, commonly assessed by clinical indicators $[1,5]$. Functional limitations are concerned with functioning of body parts whereas pain and discomfort refer to the experiential aspects of oral conditions in terms of symptoms. In addition to dissatisfaction with dental appearance, they comprise the intermediate impacts, caused by oral health status. Finally, the ultimate outcomes of disability and handicap refer to any difficulties in performing activities of daily living and to broader social disadvantages. The OIDP concentrates only on the third level of measurement and is calculated by multiplying frequency and severity scores of daily performances. As compared to using only the OIDP frequency or severity scores, applications of weighted scores revealed no significant improvement [9]. Other socio-dental indicators have also been reported to be satisfactory in terms of unweighted instead of weighted scores [10]. For those reasons, the un-weighted or abbreviated version of the OIDP frequency scale was applied in this study.

The OIDP scale has been shown to have acceptable psychometric properties when applied to adult populations in Tanzania, Thailand, UK and Greece $[4,5,11]$. In a recent study from Brazil, the OIDP scale was found to discriminate between children with and without treated dental fractures on permanent teeth [3]. One contentious finding commonly reported is the weak association found between professionally-and self-defined oral health status [12-14]. Following the propositions implicit in the theoretical discussion of oral quality of life [15], the association between clinical and subjective indicators is not direct but mediated through social, psychological and cul- tural factors. So far, however, this relationship has received only limited empirical attention [16-18].

A previous study of school going adolescents in Uganda revealed a relatively high caries experience by East African standards [19]. Thus, it was decided to supplement the clinical indicators employed to provide a more comprehensive account of their oral health status. Therefor the overall aim of this study was to assess the applicability of an abbreviated version of the OIDP questionnaire (i.e. the unweighted OIDP frequency scale) among Ugandan adolescents. Specifically, the following topics were addressed. First to assess the discriminant validity of the OIDP frequency scale by assessing it's ability to distinguish between students with and without clinically defined oral problems. Second, to assess construct validity of the OIDP frequency scale by examining it's relationship with different self-reported oral health indicators and finally to analyse the interrelationship among OIDP frequency scores, socio-demographic characteristics, oral health behaviour and clinical indicators of oral health status.

\section{Method \\ Study population and sampling procedure}

The study population was students attending public secondary schools in Kampala and Lira. Kampala is the capital city, which accounts for nearly $45 \%$ of all urban residents in Uganda. Lira district is typically rural and situated $350 \mathrm{~km}$ from Kampala. Although the proportion of the urban-rural population in Uganda is $1: 5$, approximately $80 \%$ of all secondary schools are located in urban areas. Thus, there was no strict justification for a proportional sampling of students from the two areas to reflect the urban-rural population ratio. The fluoride concentration of the drinking water in Kampala is $0.3 \mathrm{mg} / \mathrm{L}$. In Lira, the fluoride concentration in the water was unknown at the time of the survey. Water samples were subsequently collected in tight-lidded plastic bottles for fluoride analysis. The fluoride concentration in Lira ranged from 0.10 $\mathrm{mg} / \mathrm{L}$ to $1.20 \mathrm{mg} / \mathrm{L}$ with an average of $0.50 \mathrm{mg} / \mathrm{L}$ [19].

The STATA statistical program was used to estimate the sample size, allowing for a design factor, an assumed caries proportion in the Ugandan population and required precision. The sample size was calculated following the guidelines of our statistician. Briefly, using a software package that takes account of complex sample designs (STATA), and assuming a design factor of 1.7, a standard error of 0.02 and a proportion of sugar consumption of 25 $\%(\mathrm{p}=0.25)$, yielded a sample size of 1.300 students.

Twenty schools were listed in Lira and 10 considered for sampling, the inclusion criteria being schools with at least 250 students and placed at least $10 \mathrm{~km}$ from Lira town center. In Kampala, 30 schools within the radius of $10 \mathrm{~km}$ 
from the city center (main Post Office building) were considered for sampling. A total of 10 secondary schools (5 from Kampala and 5 from Lira) were selected by simple random sampling. A total of 1146 out of 1324 eligible students, 52\% urban, mean age 15.8 (SD1.6, range 13-19), response rate $87 \%$, completed structured questionnaires in the autumn of 2001. A sub-sample for a subsequent survey including a clinical examination was selected by systematic random sampling from the list of the participants of the main survey. The follow-up survey was conducted three months after completion of the main survey. A total of 372 students out of 515 sampled, 48\% urban, mean age 16.3 (SD 1.7), response rate $72 \%$, completed a short version of the original questionnaire and were examined for dental caries following the WHO diagnostic criteria [20]. Absenteeism from school was the main reason for non-response. Written informed consent to participate in the study was obtained from students and their parents/guardians. Ethical clearance to conduct the study was granted by the Ministries of Health and Education in Uganda, the local administration, school authorities and the ethical research committees in Uganda and Norway.

\section{Survey instrument}

The questionnaire was constructed and completed in English, the language of instruction in Ugandan secondary schools. This made the translation and back-translation of the questionnaire unnecessary. Sensitivity to culture and selection of appropriate words were considered. Oral health professionals and methodologists reviewed the survey instrument for semantic equivalence, experiential equivalence and conceptual equivalence. It was pilot tested before use. Some modifications concerning clarification of the content and simplification of the wording was considered necessary after the pilot study. The students completed the questionnaires in their respective classrooms under the supervision of trained research assistants and in the absence of the teacher to ensure confidentiality and to reduce response bias. The questionnaire of the main-survey had 78 questions assessing sociodemographic characteristics, the OIDP inventory, oral health related behaviours, self-assessed oral health and social and psychological factors related to intake of sugared snacks and drinks. The questionnaire of the followup survey had 32 questions. It addressed sugar intake and related socio-psychological factors a second time in addition to the main socio-demographic characteristics.

\section{Measures}

Socio-demographic characteristics were assessed in terms of gender, age and place of residence. Father's and mother's highest level of education were assessed on scales ranging from 1 = "have not gone to school" to 5 = "university". Two dummy variables were constructed yielding the categories 0 = "lower education" (including no education/pri- mary school and adult education) and $1=$ "higher education" (including secondary school, college, university) and added into a sum score of Parental education yielding the categories $0=$ low, $1=$ medium, $2=$ high. Religious groups was assessed to probe into culturally different lifestyles (e.g. eating habits) that are evident between the main religious belongings; $1=$ Catholic, $2=$ Protestant and $3=$ Muslims/ others. Thus, religious affiliation was used as a social marker in the analyses.

Oral impact of daily performance was obtained by adding scores for eight frequency items. "During the past 6 months how often have problems with your mouth and teeth caused you any difficulties with, 1) eating, 2) speaking and pronouncing clearly, 3) cleaning teeth, 4) sleeping and relaxing, 5) smiling without embarrassment, 6) maintaining emotional state, 7) enjoying contact with other people and 8) carrying out major school work. The scale used was in the range: (0) "never affected", (1) "less than once a month", (2) "once or twice a month", (3) "once or twice a week" (4) "3-4 times a week", (5) "every or nearly every day". For analysis, dummy variables were constructed yielding the categories $0=$ "never affected" (including the original category 0 ) and $1=$ "affected less than once a month or more often" (including the original categories 1-5). Simple count scores (SC) were created by adding the 8 dummy variables. Additive scores (ADD) were created by adding the 8 OIDP items as assessed originally. Finally the OIDP SC frequency scores were dichotomised, yielding the categories (0) " no daily performance affected" and (1) "at least one daily performance affected". Proportions of missing cases ranged from 0.5 (difficulty with cleaning teeth) to $2.3 \%$ (difficulty with carrying out major school work).

Received oral health care was assessed by one question: "During the past 2 years have you attended a dental clinic in order to receive treatment?" The response categories were $1=$ yes, $0=$ no. Satisfaction with dental appearance/oral condition was assessed by one item each, i.e. "Are you satisfied or dissatisfied with the appearance/condition of your teeth"? A 5-point response scale was used ranging from (1) "very satisfied" to (5) "very dissatisfied". For analyses two dummy variables were constructed yielding the categories (0) "Satisfied with dental appearance/ oral condition" and (1) "Dissatisfied with dental appearance/ oral condition". Last dental appointment painful was assessed by one item, i.e. " If you have attended a dental clinic, was your last visit painful?" using the categories (0) Not painful at all and (1) at least slightly painful

\section{Clinical examination}

One trained dentist conducted an oral examination under field conditions with an assistant recording the observations. Caries was assessed using the decayed, missing and 
filled tooth index (DMFT) as described by the World Health Organisation, WHO [20]. The child was seated on a chair in the shade outside the school building. Cotton rolls were used to clean the teeth and to control saliva. Natural light was the source of illumination. Sharp dental probes and plane mouth mirrors were employed to assess carious lesions. Except third molars, teeth with any part visible through the gum were examined. Caries was recorded as being present when a lesion in a pit or fissure or on a smooth surface had a detectable softened floor, undermined enamel, softened wall or a temporary filling. On proximal surfaces, the probe had to enter a lesion with certainty. A tooth was considered missing, if there was a history of extraction due to pain and / or the presence of a cavity.

\section{Statistical analyses}

Data were analysed using SPSS (version 10.0). Non-parametric statistics were the primary choice because the OIDP frequency scores were not normally distributed. Cohen's kappa and Cronbach's alpha was used to test for intraexaminer agreement and internal consistency reliability, respectively. To assess discriminant validity, multivariate analyses were performed by logistic regression and with the dichotomised OIDP SC scores as dependent variable, checking for all possible 2-way interactions between independent variables. GLM ANOVA was conducted to assess construct validity after using log transformation (non-linear transformation) of the OIDP ADD scores. As the clinical examination was restricted to a sub-sample, the multivariate logistic regression analyses comprised only 372 students. Age, gender, parental education, religious affiliation and place of residence were forced into multivariate analyses independent of statistical significance with the outcome variable to control for potential confounding effects. To correct for effects of the cluster design, logistic regression analyses were re-analysed with STATA (7.0) using the svylogit command. The statistically significant relationships observed initially were left essentially unchanged.

\section{Results \\ Reliability}

Cohen's kappa calculated for the socio-demographic characteristics assessed in the main and follow-up survey showed satisfactory values ranging from 0.61 to 0.91 . Approximately $10 \%(n=47)$ of the respondents from the clinical examination had their teeth re-examined clinically for dental caries after one week. The agreement was satisfactory with a Cohen's kappa of 0.82 . There was no evidence of systematic errors in the recording of dental caries as tested by Wilcoxon signed-rank test ( $\mathrm{p}>0.74)$.

Table 1 gives the percentage distribution of participants' socio-demographic characteristics separately for the total number of participants $(\mathrm{n}=1146)$ and for those of the sub-sample $(\mathrm{n}=372)$. To assess whether the participants of the sub-sample, who participated both in the main-and follow-up study were representative of the study group as a whole, a comparison was made between the former group of participants and those $(n=774)$ who completed the main survey, only. As to the socio-economic and demographic variables and the non-clinical variables utilised in this study, there were no statistically significant differences between the two groups.

The mean OIDP ADD and OIDP SC scores were respectively, $13.9(\mathrm{sd}=7.4$, range $8-40)$ and $2.7(\mathrm{sd}=2.9$, range $0-8$ ) (Table 2). A total of $44 \%$ and $30 \%$ of the participating students confirmed difficulties with eating and with showing teeth, respectively. The second most prevalent impact was difficulties with cleaning teeth $(35 \%)$, followed by difficulties with speaking and carrying out major schoolwork (34\%). The floor effect was substantial in that a total of $38 \%$ had OIDP scores of zero using both the $\mathrm{ADD}$ and the SC scoring method. A total of $62 \%$ of the students experienced at least one impact during the 6 months preceding the survey.

The inter-item correlation coefficients among the 8 OIDP items ranged from 0.42 (between eating and showing teeth) to 0.63 (between school work and social contact) (Table 3). No correlation was negative indicating homogeneity among the items and no correlation was high enough for any item to be redundant. The corrected itemtotal correlation ranged from 0.62 to 0.75 being above the minimum recommended level of 0.20 for inclusion of items in a scale and meeting the stringent criterion of item convergent validity of $>0.40$ [20]. The Cronbach's alpha of the scale was 0.90 with the alpha values if any item being deleted lower than the original value. The present alpha value falls within the recommended minimum of $0.70[20]$.

Overall DMFT scores ranged from 0.0 to 14.0 with a mean of 2.9 (SD 2.3). Moreover a total of $80 \%, 77 \%$ and $28 \%$ had respectively, DMFT $>0$, DT $>0$ and MT $>0$. Only one child had FT $>0$. In terms of discriminant validity, none of the OIDP frequency items discriminated statistically significantly between students with (DT $>0$ ) and without $(\mathrm{DT}=0)$ decayed teeth.. As shown in Table 4, statistically significant differences were observed between students with $(\mathrm{MT}>0)$ and without missing teeth $(\mathrm{MT}=0)$ across the entire range of OIDP frequency items. Students with missing teeth were 3.4 times (95\% CI 2.0-5.7) and 1.8 times (95\% CI 1.1-3.1) more likely to report respectively, difficulties with sleeping and relaxing and difficulties with speaking than were their counterparts without missing teeth. In terms of construct validity, statistically significant relationships were observed between the OIDP (ultimate 
Table I: Socio-economic characteristics of total number of participants $(n=1 / 46)$ and the participants of the sub-sample $(n=372)$.

\begin{tabular}{lll}
\hline & Total sample & Sub-sample \\
\hline & $\mathrm{N}=1146 \%(\mathrm{n})$ & $\mathrm{N}=372 \%(\mathrm{n})$ \\
\hline $\begin{array}{l}\text { Place of school } \\
\text { Kampala (urban) }\end{array}$ & $51(591)$ & \\
Lira (rural) & $49(555)$ & $58(180)$ \\
Gender & & $52(192)$ \\
Male & $60(667)$ & $53(202)$ \\
Female & $40(450)$ & $47(181)$ \\
Age & $45(515)$ & $34(131)$ \\
I3-15 & $55(631)$ & $66(253)$ \\
16-19 & $56(630)$ & $56(206)$ \\
Dental attendance & $44(159)$ \\
No & $44(496)$ & $28(105)$ \\
Yes & & $37(135)$ \\
Parental education & $28(321)$ & $35(130)$ \\
Low & $31(352)$ & $32(119)$ \\
Medium & $4 I(454)$ & $47(174)$ \\
High & $33(376)$ & $21(77)$ \\
Religious affiliation & $44(504)$ & \\
Catholic & $23(256)$ & \\
Protestant & & \\
Muslims/others & & \\
\hline
\end{tabular}

The total number in the different categories do not add up to II 46 and 372 due to missing values

Table 2: Percentage distribution (percentages of students affected less than once a month or more) and mean frequency scores (SD) for the eight OIDP items and the OIDP ADD and OIDP SC scores (n ranging from I I 22-I I40)

\begin{tabular}{lll}
\hline & \% affected $(\mathrm{N})$ & Mean score $(\mathrm{I}-5)(\mathrm{SD})$ \\
\hline I. Eating & $44(494)$ & $1.9(1.3)$ \\
2. Speaking & $34(387)$ & $1.7(1.2)$ \\
3. Cleaning teeth & $35(398)$ & $1.8(1.3)$ \\
4. Sleeping /relaxing & $33(379)$ & $1.7(1.2)$ \\
5. Showing teeth & $30(350)$ & $1.7(1.2)$ \\
6. Emotional status & $33(375)$ & $1.7(1.2)$ \\
7. Carrying out work & $34(386)$ & $1.7(1.2)$ \\
8 Enjoy social contact & $31(356)$ & $1.6(1.1)$ \\
Total OIDP SC scores & $62(671)$ & $2.7(2.9)$ \\
Total OIDP ADD scores & - & $13.9(7.4)$ \\
\hline
\end{tabular}

a percentages of students affected on at least one daily performance

oral impacts) and a count of non-clinical oral health indicators representing the second (intermediate) levels of oral impacts (Table 5). The more impacts experienced the higher the score of the OIDP ADD and OIDP SC frequency indices. Students being dissatisfied with dental appearance and oral condition and those who experienced last dental visit as painful scored significantly higher on the OIDP than did their peers in the opposite groups.
Rural students scored higher than their urban counterparts (mean OIDP SC: 3.0 versus 2.3, p < 0.001) and Catholics scored higher than Muslims (mean OIDP SC: 3.0 versus 2.4, $\mathrm{p}<0.05$ ). Moreover, students who confirmed dental attendance scored significantly higher on the OIDP scores than did students who had not visited a dentist (mean OIDP SC: 3.0 versus 2.4, p < 0.05). Multiple logistic regression analysis revealed that place of residence, religious affiliation and dental attendance entered in step one accounted for $9 \%$ points (Nagelkerke's $\mathrm{R}^{2}=0.093$, 
Table 3: Correlation matrix for OIDP frequency scores (I-8)

\begin{tabular}{|c|c|c|c|c|c|c|c|c|}
\hline & 1 & 2 & 3 & 4 & 5 & 6 & 7 & 8 \\
\hline I. Eating & 1.00 & & & & & & & \\
\hline 2. Speaking & .54 & 1.00 & & & & & & \\
\hline 3. Cleaning teeth & .51 & .44 & 1.00 & & & & & \\
\hline 4. Sleeping/relaxing & .58 & .53 & .54 & 1.00 & & & & \\
\hline 5. Showing teeth & .42 & .45 & .46 & .56 & 1.00 & & & \\
\hline 6. Emotion & .49 & .44 & .50 & .59 & .55 & 1.00 & & \\
\hline 7. Work & .42 & .48 & .47 & .60 & .57 & .61 & 1.00 & \\
\hline 8 Social contact & .44 & .48 & .50 & .60 & .61 & .55 & .63 & 1.00 \\
\hline
\end{tabular}

Table 4: Discriminant validity: Percentage distribution ( $n$ ) and odds ratio of single and overall OIDP SC frequency scores by clinical indicator $(n=372)$. (OR adjusted for age, gender, place of residence, religious affiliation, parental education).

\begin{tabular}{lllll}
\hline & $M T>0 \%(n)$ & $M T=0 \%(n)$ & Unadjusted OR $(95 \% \mathrm{Cl})^{\mathrm{a}}$ & Adjusted OR $(95 \% \mathrm{Cl})^{\mathrm{a}}$ \\
\hline Eating & $64(66)$ & $34(85)^{* *}$ & $3.4(2.1-5.5)$ & $3.3(2.0-5.5)$ \\
Speaking & $44(46)$ & $29(74)^{*}$ & $1.9(1.2-3.1)$ & $1.8(1.1-3.1)$ \\
Cleaning teeth & $51(53)$ & $26(70)^{* *}$ & $2.8(1.7-4.5)$ & $2.7(1.6-4.6)$ \\
Sleeping and relaxing & $51(52)$ & $22(58)^{* *}$ & $3.5(2.1-5.7)$ & $3.4(2.0-5.7)$ \\
Smiling and showing teeth & $36(68)$ & $26(68)^{*}$ & $1.7(1.0-2.7)$ & $1.6(1.0-2.8)$ \\
Emotional status & $45(46)$ & $26(68)^{*}$ & $2.2(1.3 .3 .6)$ & $2.1(1.3-3.5)$ \\
School work and social role & $43(44)$ & $28(70)^{*}$ & $2.0(1.2-3.2)$ & $2.0(1.2-3.3)$ \\
Enjoying social contact & $49(49)$ & $25(64)^{* *}$ & $2.8(1.7-4.5)$ & $2.7(1.6-4.5)$ \\
OIDP SC score $>0$ & $74(74)$ & $56(135)^{* *}$ & $2.2(1.3-3.7)$ & $2.2(1.3-3.8)$ \\
\hline
\end{tabular}

$*_{\mathrm{P}}<0.05,{ }^{*} \mathrm{p}<0.00 \mathrm{I}$ a Reference category $(\mathrm{I}):$ OIDP $=0$

Table 5: Construct validity: Mean values and $95 \% \mathrm{Cl}$ (confidence interval) for OIDP SC and OIDP ADD scores by subjective oral health indicators $(n=1$ I 46) (Adjusted for age, gender, place of residence, religious affiliation and parental education).

\begin{tabular}{lll}
\hline & OIDP ADD scores & OIDP SC scores \\
\hline $\begin{array}{l}\text { Subjective oral health indicators } \\
\text { Dental appearance }\end{array}$ & & \\
Good $(\mathrm{n}=746)$ & $12.2(11.6-12.7)$ & $2.1(1.8-2.3)$ \\
Bad $(\mathrm{n}=315)$ & $17.8(16.8-18.5)^{* * *}$ & $4.1(3.7-4.3)^{* *}$ \\
Dental condition & & $2.1(1.9-2.3)$ \\
Good $(\mathrm{n}=760)$ & $12.3(11.8-12.8)$ & $4.1(3.7-4.3)^{* *}$ \\
Bad $(\mathrm{n}=302)$ & $17.9(16.9-18.64)^{* *}$ & $2.1(1.8-2.4)$ \\
Last dental appointment & $12.7(11.8-13.5)$ & $3.8(3.4-4.0)^{* *}$ \\
Not painful $(\mathrm{n}=321)$ & $16.2(15.4-16.9)^{* *}$ & \\
Painful $(\mathrm{n}=463)$ &
\end{tabular}

$*^{*} \mathrm{p}<0.001, * \mathrm{p}<0.05$

Model chi-square 23.674, $\mathrm{df}=4, \mathrm{p}<0.001$, percentage correctly classified $62.7 \%$ ) of the explainable variance in the OIDP SC scores. Entering the clinical indicator raised the explainable variance by a modest $2 \%$ points to bring it to a final $11 \%$ points (Nagelkerke's $\mathrm{R}^{2}=0.114$, Model chi square $=29.243, \mathrm{df}=6, \mathrm{p}<0.001$, percentage correctly classified $66.17 \%$ ). In the final model, it was shown that Muslims were less likely to score OIDP > 0 compared to those with Catholic affiliation. Students having attended a dentist and students with MT $>0$ were more likely to score OIDO $>0$ compared to their counterparts. As shown in Table 6 , the odds ratios were respectively $0.5(0.3-0.9)$, 
Table 6: Odds ratios (OR) and $95 \%$ confidence interval $(\mathrm{Cl})$ for respondents' OIDPS SC scores $(0=$ no impacts, OIDP $>0=I)$ by socio-demographic characteristics, dental attendance and clinical status (adjusted for age, gender parental education).

\begin{tabular}{|c|c|c|c|c|}
\hline & \multicolumn{2}{|c|}{ Unadjusted } & \multicolumn{2}{|c|}{ Adjusted } \\
\hline & OR & $95 \% \mathrm{Cl}$ & OR & $95 \% \mathrm{Cl}$ \\
\hline \multicolumn{5}{|c|}{ Block I } \\
\hline \multicolumn{5}{|c|}{ Place of residence } \\
\hline Urban & 1 & & I & \\
\hline Rural & 1.3 & $1.1-1.7$ & 1.0 & $0.5-1.6$ \\
\hline \multicolumn{5}{|c|}{ Religious affiliation } \\
\hline Catholic & 1 & & I & \\
\hline Protestant & 0.7 & $0.5-0.9$ & 0.6 & $0.3-1.1$ \\
\hline Muslim & 0.5 & $0.3-0.8$ & 0.5 & $0.3-0.9$ \\
\hline \multicolumn{5}{|c|}{ Dental attendance } \\
\hline No & I & & I & \\
\hline Yes & 2.0 & $1.5-3.5$ & 2.2 & $1.3-3.5$ \\
\hline \multicolumn{5}{|c|}{ Block 2} \\
\hline \multicolumn{5}{|c|}{ Missing teeth (MT) } \\
\hline $\mathrm{MT}=0$ & 1 & & I & \\
\hline MT $>0$ & 2.2 & $1.3-3.7$ & 1.8 & $1.0-3.2$ \\
\hline
\end{tabular}

$2.2(1.3-3.5)$ and $1.8(1.0-3.2)$. When adjusting for confounding variables in terms of socio-demographics, nonclinical and clinical variables, place of residence did not maintain its statistically significant relationship. Rather its effect was mediated by non-clinical and clinical variables. No statistically significant interaction effect between MT and social and behavioural variables was identified. However, one statistically significant second order effect occurred for gender by place of school $(\mathrm{B}=1.4, \mathrm{p}<0.001)$. Among males reporting at least one oral impact was equally common in Kampala, 61\% and Lira 64\% (p > $0.05)$. For females the corresponding figures were $57 \%$ and $71 \%(\mathrm{p}<0.001)$, respectively.

\section{Discussion}

Cross-cultural adaptation of socio-dental indicators requires rigorous translation and validation to make the adopted instrument culturally relevant for the local population [21-23]. The participants of the present study were familiar with English as a second mother tongue, thus a rigorous translation and back-translation was deemed unnecessary. To ensure interpretability and cultural equivalence, the OIDP was pre-tested among the students and supervised by a group of Ugandan researchers. This process concluded with a minor rephrasing of the question about major work and social role, which was modified into "carrying out major schoolwork".

The participants of this study attended public secondary schools, randomly selected from the population of secondary schools in Kampala and Lira. Thus, they might have captured the variety of characteristics of 13-19 yearold adolescents attending secondary schools in those areas. Since attendance is voluntary and many students leave school early for different reasons, the participants are not representative of the general population of youth at similar ages in Kampala and Lira. Due to realities of life in Uganda, general youth population surveys including out of school adolescents are difficult to conduct and even the national oral health surveys have been confined to school going children and adolescents [24].

A total of $62 \%$ of the participants reported experiencing an oral impact that affected their daily life in the past six months. The eight impact prevalence rates ranged from $30 \%$ to $44 \%$ and the floor effect of $32 \%$ indicates that the OIDP impacts are of relatively high severity in this study population. Consistent with the results reported in previous OIDP surveys [3-6], difficulty with eating and enjoying food and with cleaning teeth were the impacts most frequently reported. The total impact prevalence rate was comparable with, but slightly higher than that of $51 \%$, observed in the Tanzanian study, using similar methodology and the English version of the questionnaire [5]. It was below those of about $70 \%$ observed in western populations with high dental disease levels and in a low-oral disease Thai population $[1,5]$. Moreover, the single OIDP prevalence rates tended to be larger in magnitude among Ugandan students as compared to their Tanzanian counterparts. With respect to their ranks within the OIDP inventory there appeared to be a reasonably degree of consistency across the two East African cultures. Variety in the results of studies using similar methods might be attributed to different disease levels and sample distributions. Alternatively, the variety in perceived oral impacts might be cultural in origin.

The OIDP frequency scores were applicable across age and gender, showed satisfactory reliability and were subject to low levels of non-response. Accordingly, Masalu and Åstrøm [5,17] found younger students and females to be as likely as their older-and male student counterparts to report impacts of oral disorders. The OIDP frequency score showed item-to-scale correlations that are similar to those obtained in previous applications [11] and the internal consistency reliability in terms of a Cronbach's alpha of 0.90 indicates excellent psychometric properties if the recommended level of 0.70 is used [21]. Previous applications of the OIDP scale to various populations have yielded internal consistency values ranging from 0.67 to $0.85[4,5,11]$. Consistent with the results reported by Masalu and Åstrøm [5] and by Tsakos et al [11], but at odds with those provided by other researchers [25], the present data revealed a negative gradient with respect to dental attendance, the more frequent this habit, the less favourable the OIDP ratings. It is plausible that dental 
attendance patterns reflect perceived treatment need among Ugandan school children and might be recognised as a proxy for oral health related quality of life [10]. Thus, the highest OIDP and dental attendance rates were found in Lira having a free of charge public oral health service, whereas the caries prevalence (DMFT $>0$ ) was highest in Kampala where cost sharing is currently implemented [19]. Recent studies from other African countries have documented an increase in patient attendance and dental operator load following the introduction of free primary dental health care [26].

Hypotheses regarding the construct validity were confirmed in that the OIDP frequency scores varied positively with global measures of self-rated oral health status, dental attendance and experience with dental pain. In terms of discriminant validity, students with missing teeth were more likely to report problems with eating, cleaning teeth and with sleeping and relaxing. Less differences were found with respect to "smiling and showing teeth without embarrassment", suggesting that Ugandan adolescents are more concerned with function than with appearance. This might be attributed to the fact that the second and first molars are the teeth most frequently affected by caries in the present study population [19]. Not unexpectedly, these findings are at odds with previous results on the impact of malocclusions and fractured incisors where dissatisfaction with dental appearance has been identified as the most important impact [3].

Although the amount of explained variance was low, suggesting omission of important variables, social and behavioural variables accounted for three times as much (9\%) of the variance in the OIDP than did the MT scores (2\%). In fact dental attendance was the strongest predictor of oral impacts, supporting its suggested role as a proxy for the ultimate oral impacts [11]. Place of residence operated as a direct antecedent of oral impacts, but only among girls. There was no evidence of a role of socio-demographic and behavioural characteristics in modifying the relationship between clinical status and OIDP, indicating a linear relationship between impairments and the ultimate oral impacts. Nevertheless, the present results confirmed all together the hypothesis that independent of the actual level of oral disease, perceived oral health status is shaped by previous experience of oral behaviours and by the prevailing social circumstances.

The present study suggests that an abbreviated OIDP inventory is applicable for use among adolescents attending secondary school in Uganda. The item level characteristics, the reliability and the fact that the OIDP scale discriminated between groups known to differ with respect to oral health add to our confidence that the scale measure a construct comparable with the original. More- over, the present study indicates that the social and behavioural context is important in shaping adolescents' responses to oral disorders. This study constitutes a first step to highlight the limits of focusing exclusive on normative needs and suggests the incorporation of oral quality of life measures into the oral health care services for the younger generation in Uganda.

\section{Competing interests}

Professor Aubrey Sheiham is co-author of the OIDP measure used in this article.

\section{Authors' contribution}

Anne Nordrehaug Åstrøm conceived of this study, determined it's design, conducted the statistical analyses and was responsible for the completion of the whole manuscript. Isaac Okullo was responsible for the data collection in Uganda, participated in the design coordination of the study and has contributed substantially to the completion of the method section. Both authors read and approved the final manuscript.

\section{Acknowledgement}

This study was supported economically by the University of Bergen. We would like to thank all secondary school students who took their time to participate in the large scale oral health survey. The assistance from Dr. Charles Rwenyonyi and Dr. Louis Muwazi at Makerere University is highly appreciated.

\section{References}

I. Slade GD: Measuring oral health and quality of life. Chapel Hill: University of North Carolina, Dental Ecology 1997.

2. Buck D and Newton JT: Non-clinical outcome measures in dentistry: publishing trends 1988-98. Community Dent Oral Epidemiol 200I, 29:2-8.

3. Cortes MIS, Marcenes W and Sheiham A: Impact of traumatic injuries to the permanent teeth on the oral health related quality of life in I2-14 year old children. Community Dent Oral Epidemiol 2002, 30: 193-8.

4. Masalu J and Åstrøm AN: Applicability of an abbreviated version of the oral impacts on daily performances (OIDP) scale for use among Tanzanian students. Community Dent Oral Epidemiol 2003, 3 I:7-14.

5. Adulyanon S, Vourapukjaru J and Sheiham A: Oral impacts affecting daily performance in a low dental disease Thai population. Community Dent Oral Epidemiol 1996, 24:385-9.

6. Tsakos G, Marcenes W and Sheiham A: Cross-cultural differences in oral impacts on daily performance between Greek and British older adults. Community Dent Health 200 I, I 8:209-2 I3.

7. Badley EM: The ICIDH: format, application in different settings and distinction between disability and handicap. Int Disabil Stud 1987, 9:122-5.

8. Locker D: Measuring oral health: a conceptual framework. Community Dent Oral Epidemiol 1988, 5:3-18.

9. Adulyanon $S$ and Sheiham A: Oral Impacts on Daily Performances. In: Measuring oral health and quality of life. Edited by: Slade GD. Chapel Hill: University of North Carolinas; 1997.

10. Allen PF and Locker D: Do item weights matter? An assessment using the oral health impact profile. Community Dent Health 1997, 25:284-90.

II. Tsakos G, Marcenes W and Sheiham A: Evaluation of a modified version of the index of oral impacts on Daily performances (OIDP) in elderly populations in two European countries. Gerodontology 200I, I 8:121-130. 
12. Leao $A$ and Sheiham A: Relation between clinical dental status and subjective impacts on daily living. J Dent Res 1995, 74:1408-13.

13. Locker D: Clinical correlates of changes in self-perceived oral health in older adults. Community Dent Oral Epidemiol 1997, 25:199-203.

14. Locker D and Slade G: Association between clinical and subjective indicators of oral health status in an older adult population. Gerodontology I994, I I:I08- I4.

15. Chen MS and Hunter P: Oral health and quality of life in New Zealand: a social perspective. Soc Sci Med 1996, 43:1213-22.

16. Locker D: Health outcomes of oral disorders. Int J Epidemiol 1995, 24:85-9.

17. Masalu J and Åstrøm AN: Social and behavioral correlates of oral quality of life studied among university students in Tanzania. Acta Odontol Scand 2002, 60:353-359.

18. Newton JT, Robinson PG, Khan F, Gelbier S and Goibbons DE: Testing a model of the relationship between gender, ethnicity, clinical status and impact in older adults from minority ethnic groups. Gerodontology 2002, 19:102-108.

19. Okullo I, Åstrøm AN, Haugejorden O and Rwenyonyi CM: Variation in caries experience and sugar intake among secondary school students in urban and rural Uganda. Acta Odontol Scand 2003.

20. World Health Organisation: Oral health surveys. Basic methods. Geneva 1997.

21. Mc Dowell I and Newell C: Measuring Health. A guide to rating scales and questionnaires. New York: Oxford University Press II 996.

22. Allison P, Locker D, Jokovic A and Slade G: A cross-cultural study of oral health values. J Dent Res 1999, 78:643-9.

23. Beaton DE, Bombardier CD, Guillemin F and Ferraz MB: Guidelines of the process of cross-cultural adaptation of self-report measures. Spine 2000, 25:|86-9|.

24. Tirwomwe F, Ekoku Y, Manji F, Bælum V and Fejerskov O: Oral health in Uganda: results from a national survey 1987. Ministry of Health, Uganda/Kenya. Medical Research Institute 1988.

25. Unell L, Søderfeldt B, Halling A and Birkhed D: Explanatory models for oral health expressed as number of remaining teeth in an adults population. Community Dent Health 1998, 15:155-61.

26. Bhayat $A$ and Cleaton-Jones $P$ : Dental attendance in Soweto, South Africa, before and after the introduction of free primary dental health services. Community Dent Oral Epidemiol 2003 3I:I05-I0.

\section{Pre-publication history}

The pre-publication history for this paper can be accessed here:

http://www.biomedcentral.com/1472-6831/3/5/prepub
Publish with Bio Med Central and every scientist can read your work free of charge

"BioMed Central will be the most significant development for disseminating the results of biomedical research in our lifetime. "

Sir Paul Nurse, Cancer Research UK

Your research papers will be:

- available free of charge to the entire biomedical community

- peer reviewed and published immediately upon acceptance

- cited in PubMed and archived on PubMed Central

- yours - you keep the copyright
BioMedcentral 